\title{
Cyclophosphamide/Fluorouracil/Fluoxymesterone/Metho Regimen
}

National Cancer Institute

\section{Source}

National Cancer Institute.

Cyclophosphamide/Fluorouracil/Fluoxymesterone/Methotrexate Regimen. NCI

Thesaurus. Code C10214.

A chemotherapy regimen consisting of cyclophosphamide, methotrexate, fluorouracil, and fluoxymesterone that may be used as maintenance therapy for breast cancer. 